\title{
Monte Carlo simulations of $n$-butane and $n$-octane adsorbed onto graphite and a molecular model of activated carbon
}

\author{
María E. Farías Hermosilla ${ }^{1}$. Néstor A. Pérez Chávez ${ }^{1}$. Alberto G. Albesa ${ }^{1}$
}

Received: 27 June 2018 / Revised: 23 April 2019 / Accepted: 25 April 2019

(c) Springer Science+Business Media, LLC, part of Springer Nature 2019

\begin{abstract}
In this work we report results of Monte Carlo simulations of n-butane and $n$-octane adsorbed onto graphite and a molecular model of activated carbon, with the aim to provide simplified models that will allow the study of these kind of systems with lower computational and time costs. A combination of Coarse Grain models (CG) and Monte Carlo simulations (MC) were used. Adsorbates were reduced from four atoms to one pseudoatom and the adsorbent carbons from two atoms to one pseudoatom. We compare the results between atomistic models results and CG models for $n$-alkanes (single and mixture) adsorption on carbon surfaces, and we took into account the isosteric heat too. The simulation results showed an excellent agreement with the atomistic model for $n$-alkanes adsorption on graphite, using the CG model. However, for the activated carbons studied there is no longer such a good agreement between atomistic models and coarse-grained models.
\end{abstract}

Keywords Coarse grain models $\cdot$ Graphite $\cdot$ Activated carbon $\cdot$ Alkanes

\section{Introduction}

Adsorption phenomena were widely studied for their potential impact on environmental remediation and separation of compounds. Alkane adsorption is particularly important to storage gases. In the last years there had been a great interest in the study of alkane adsorption on carbonaceous materials, mainly in activated carbons (Fletcher and Thomas 1999; Foley et al. 2001; Fletcher et al. 2006; Severson and Snurr 2007; Liu et al. 2017).

Activated carbons are disordered materials and cannot be characterized completely from experiments, therefore realistic models of activated carbons are necessary to predict the properties of adsorbed phases (Albesa et al. 2012a).

It is important to take into account that, to study the adsorption of any substance on carbonaceous materials, studying the adsorption of these substances on graphite

Electronic supplementary material The online version of this article (https://doi.org/10.1007/s10450-019-00104-7) contains supplementary material, which is available to authorized users.

Alberto G. Albesa

albesa@inifta.unlp.edu.ar

INIFTA (Depto. de Química, Fac. Cs. Exactas, UNLP, CICPBA, CONICET), Casilla de Correo 16, Sucursal 4, B1904DPI La Plata, Argentina should be the first step since it is a surface that can be characterized and serves as a zero-order approximation to study adsorption on more complex materials (Albesa et al. 2008).

In recent decades, computer simulations have become an indispensable tool for studying molecules in confined spaces. The increase in computational speed and better molecular modeling algorithms allow simulations of larger systems with greater precision. The need to achieve larger simulated time scales and system sizes requires further simplification of atomistic models, even those based on empirical potentials. Recently several approaches called "coarse grain" (CG) have been developed with that goal in mind. The philosophy of these CG approaches is generally the same: to achieve a simpler description of the effective interactions in a given system without losing the ability of the resulting models to predict the properties of interest (Chiu et al. 2010; Shelley et al. 2001; Maerzke and Siepmann 2001; Mognetti et al. 2009; Gobbo et al. 2013; Shang et al. 2017; Jiang et al. 2017).

In the present work we will study the adsorption of $n$-butane and $n$-octane on graphite and activated carbons using two models for the alkane molecules and two models for the carbon atoms of the adsorbents. 


\section{Methodology}

In the present work, aggregates of polyaromatic molecules containing 24, 54, 96 and 150 carbon atoms are used to simulate the surface (Albesa 2010).

Monte Carlo simulations were performed using the grand canonical ensemble $(\mu, \mathrm{V}, \mathrm{T})$, since the number of molecules is not a fixed variable, information can be obtained about the average number of particles in the system as a function of external conditions. In this ensemble, in addition to the fact that the particles present in the system can be moved and evaluated, according to the acceptance rule of Eq. (1), particles are also created and destroyed in the system with acceptance probabilities are given by Eqs. (2) and (3) respectively;

$$
\begin{aligned}
& P_{\text {mov }}=\min \left\{1, \exp \left(-\frac{\Delta E}{k T}\right)\right\} \\
& P_{\text {cre }}=\min \left\{1, \frac{1}{(N+1)} \exp \left(B-\frac{\Delta E}{k T}\right)\right\} \\
& P_{\text {des }}=\min \left\{1, N \exp \left(\frac{\Delta E}{k T}-B\right)\right\} \\
& B=\ln \left(\frac{f V}{k_{B} T}\right)
\end{aligned}
$$

where $\mathrm{P}_{\text {cre }}$ is the probability of accepting the creation step, $\mathrm{P}_{\text {des }}$ is the probability of accepting the destruction of a particle, and $\mathrm{V}$ is the volume of the system, $\mathrm{N}$ the number of particles. The considerations and parameters taken into account in the simulations were:

(i) $10 \sigma$ cubic simulation box

(ii) Cut-off radius of $4 \sigma$;

(iii) Number of thermalization steps creation/destruction/ displacement $2.5 \times 10^{7}$, number of steps for the statistical average $5.0 \times 10^{4}$;

(iv) No long-range corrections were made due to the cutoff radio

Another advantage of the GCMC method is that information about isosteric heat can also be obtained. (Albesa et al. 2012b).

The potential energy of interaction between two particles is calculated using the well known 12-6 Lennard-Jones potential (Eq 5).

$U_{i j}=4 \varepsilon_{i j}\left[\left(\frac{\sigma_{i j}}{r}\right)^{12}-\left(\frac{\sigma_{i j}}{r}\right)^{6}\right]$

where $r$ is the separation distance between the two particles.
Table 1 - Lennard-Jones parameters

\begin{tabular}{lrl}
\hline & $\varepsilon / \mathrm{kT}$ & $\sigma(\mathrm{A})$ \\
\hline C4H10 M8 & 350 & 5.2 \\
C4H10 M1 & 421 & 4.3 \\
CH2 & 46 & 3.95 \\
CH3 & 98 & 3.75 \\
C (graphite) & 28 & 3.4 \\
C (CG) & 56 & 4.3 \\
\hline
\end{tabular}

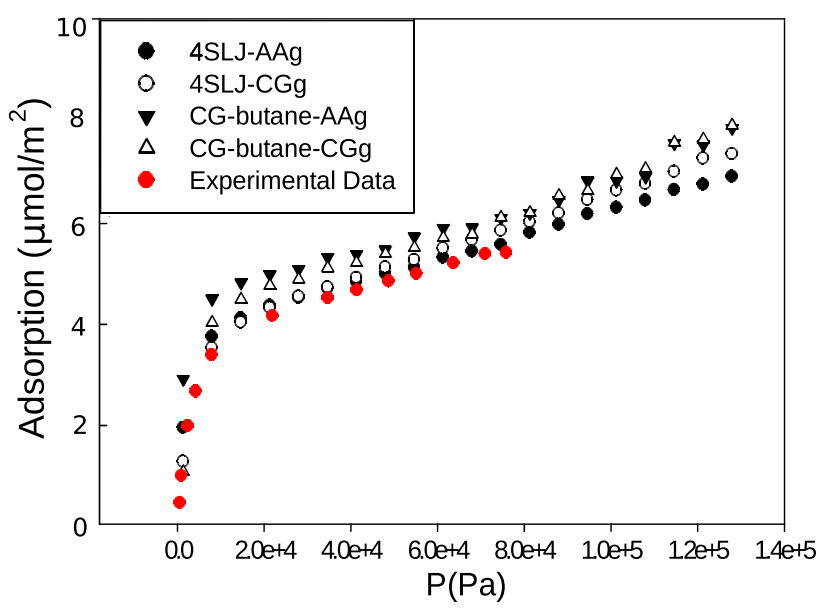

Fig. 1 Butane adsorption isotherms with the different models. $A A g$ all atom graphite, $C G g$ coarse grained graphite

The LJ parameters are listed in Table 1.

Martin and Siepmann parameters were used to simulate $n$-butane and $n$-octane with the atomistic model (Martin and Siepmann 1998).

For coarse-grain models, a 4-to-1 reduction was made for butane and octane, that is, four $\mathrm{C}$ atoms are represented by a single interaction site. For the coarse grain modeling of the graphite, the 2-to-1 reduction was used (Gobbo et al. 2013).

The interaction energy between a fluid particle and a carbon atom is also calculated by the LJ potential. The total energy is calculated by adding all the interactions of pairs between the particles of the fluid, and between the individual carbon atoms and the particles of the fluid.

\section{Results}

\subsection{Adsorption on graphite}

Figure 1 shows the adsorption isotherms for butane with the different models considered in the present work. There is an excellent agreement among all of them, showing that the new parametrization of CG-butane is good and that it not only agrees with the CG graphite model but can also be used in the completely atomistic graphite model. Likewise, 
Fig. 2 Snaphots of a adsorption of 4SLJ-butane, $\mathbf{b}$ adsorption of CG-butane
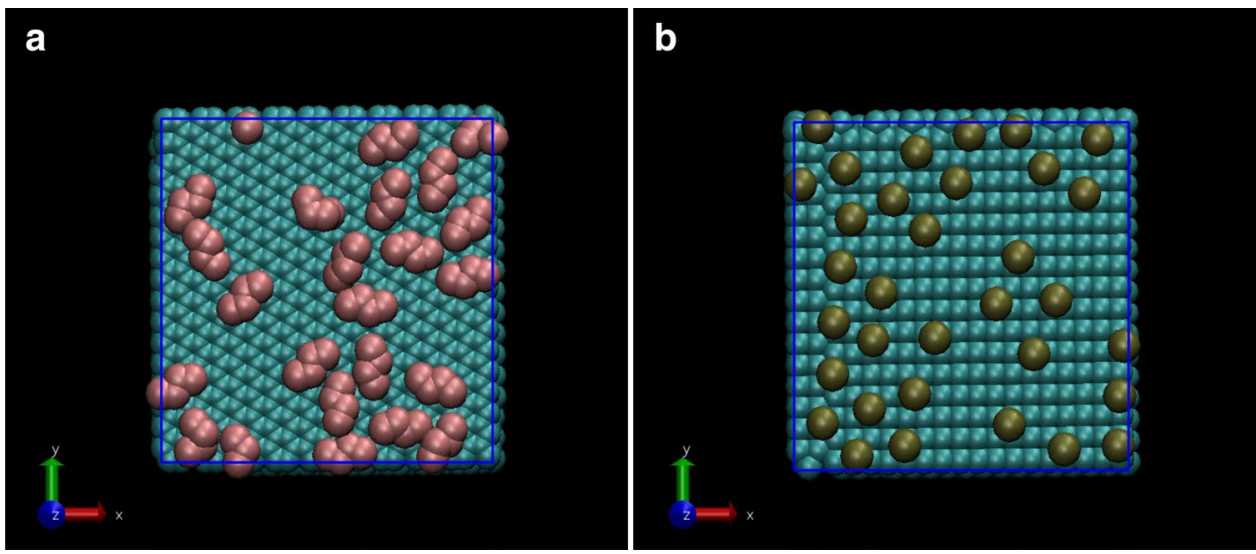

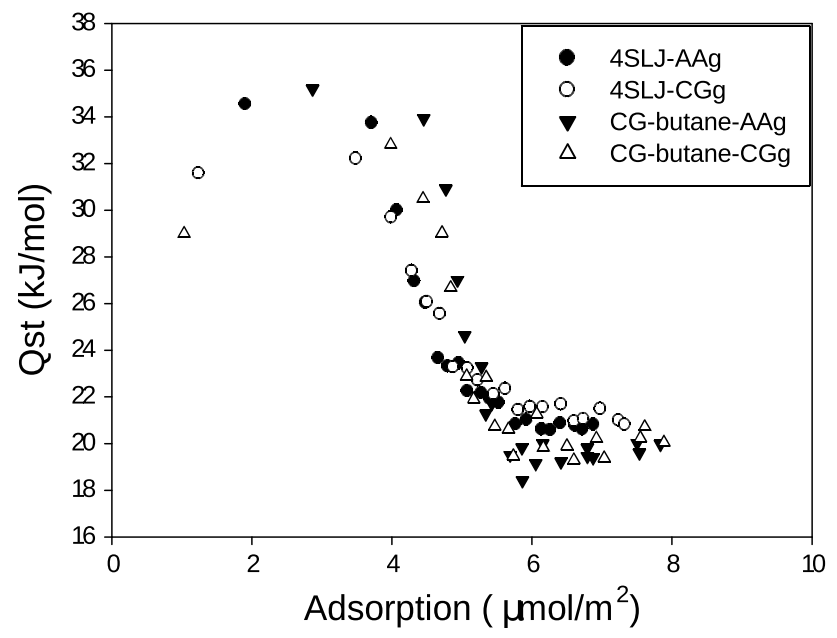

Fig. 3 Isosteric heat of adsorption of butane onto graphite

the $\mathrm{C}$ bead used in the coarse grain model also shows good agreement with the experimental results (Ross and Good 1956) when used in the 4-site butane model.

The difference observed is mainly due to the shape of the adsorbed molecules, the CG model, being completely spherical allows a more compact packing, which results in a better accommodation of the particles on the surface of graphite. As seen in Fig. 2, most of $n$-butane molecules adopt the configuration parallel to the surface and have the flat zigzag arrangement. We see from the adsorption isotherms at $303 \mathrm{~K}$ that there are no 2D transitions in the formation of the first layer.

Figure 3 shows the results of the isosteric heats of adsorption obtained in the simulations, these results show a good agreement with those obtained in the literature (Chirnside and Pope 1964).

The isotherms of Fig. 4 correspond to the adsorption of $n$-octane on graphite at $400 \mathrm{~K}$, and in Fig. 5 the values obtained for the isosteric heats are shown. The agreement between the 8 -site model and the coarse grain model of two sites is better

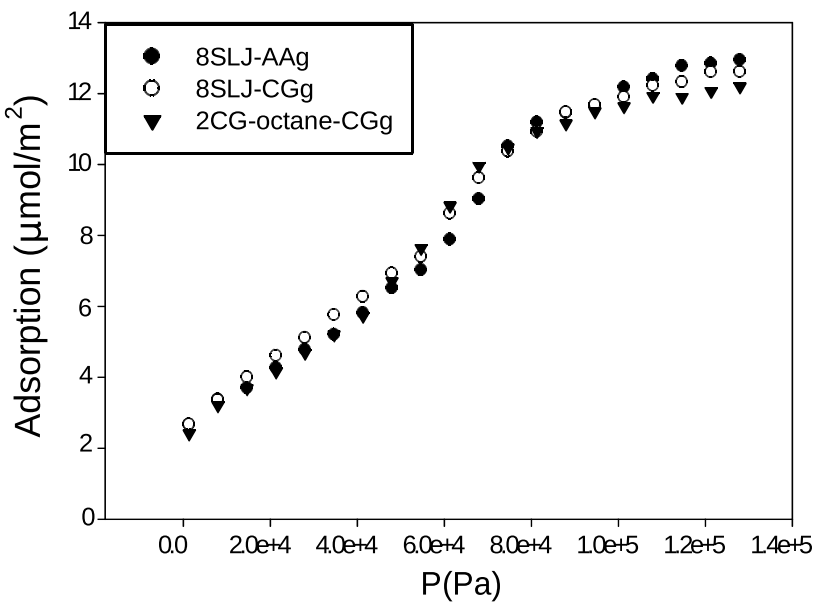

Fig. 4 Octane adsorption isotherms with the different models. $A A g$ all atom graphite, $C G g$ coarse grained graphite

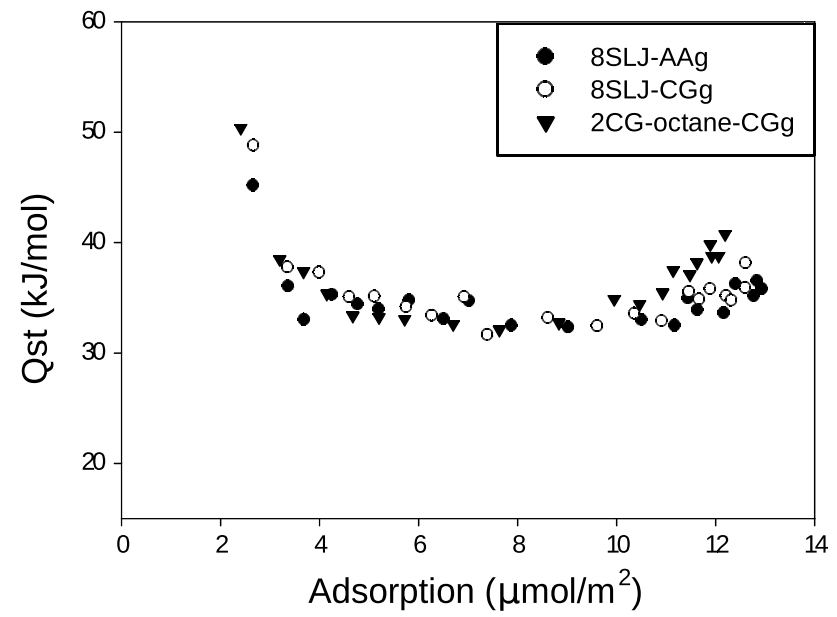

Fig. 5 Isosteric heat of adsorption of octane onto graphite 
Fig. 6 Snaphots of a adsorption of 4SLJ-octane, $\mathbf{b}$ adsorption of CG-octane
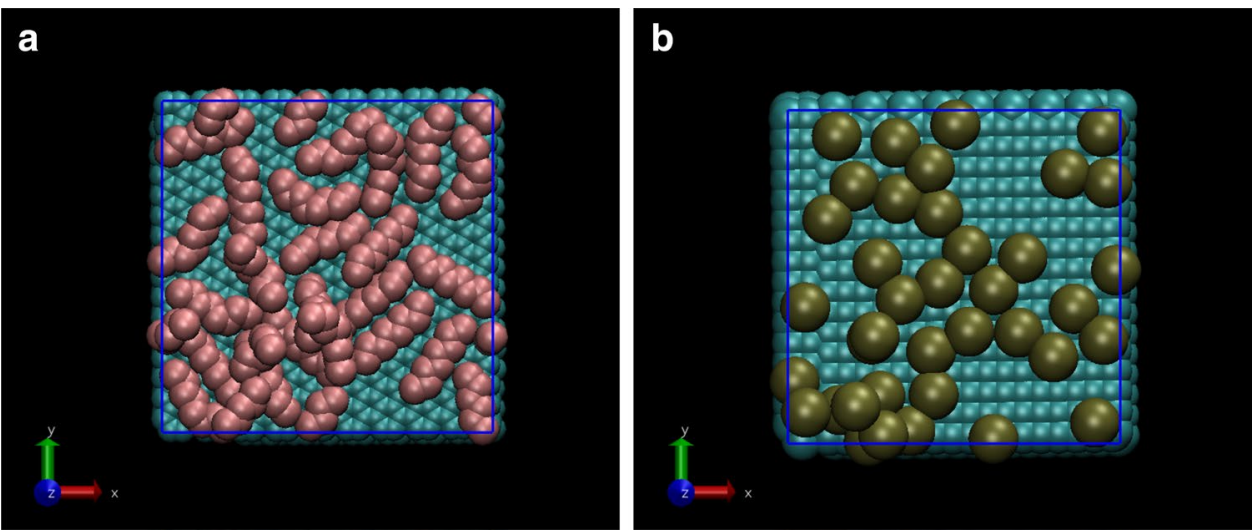

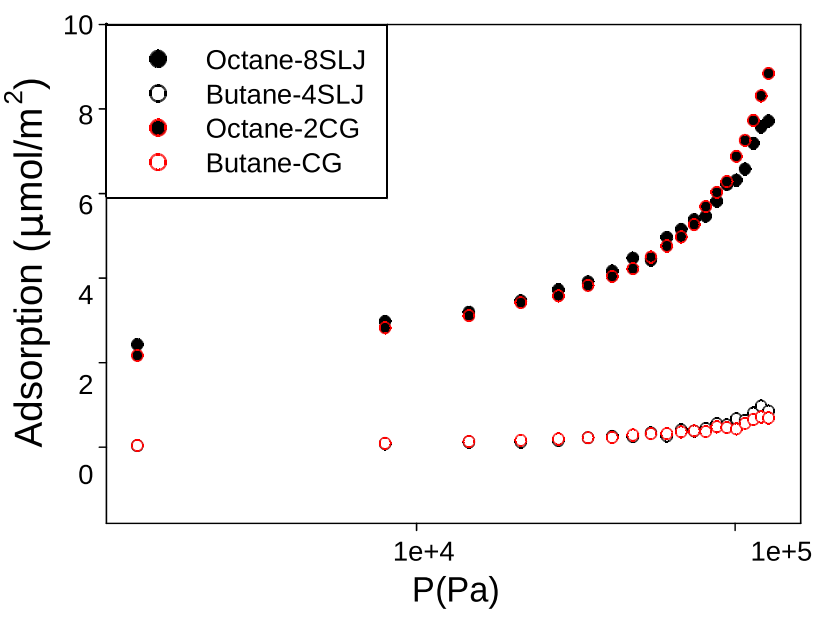

Fig. 7 Mixture adsorption isotherms with the different models. $A A g$ all atom graphite, $C G g$ coarse grained graphite

even than in the case of $n$-butane. This is explained by the fact that, in this case, the coarse-grained model of two sites already has a form factor incorporated, since by having two interaction sites separated from one another, the orientation of the molecules begins to play an important role. In Fig. 6 it is observed that the preferred orientation is parallel to the surface. The isosteric heats agree with those obtained experimentally (Eisen et al. 1971; Elkington and Curthoys 1969).

\subsection{Mixture gas adsorption}

Adsorption simulations of equimolar mixtures of $n$-octane and $n$-butane at $400 \mathrm{~K}$ were performed on graphite. In Fig. 7 the results of the isotherm are shown.

The selectivity of the species is written as

$S_{i j}=\frac{\left(n_{i} / n_{j}\right)}{\left(x_{i} / x_{j}\right)}$

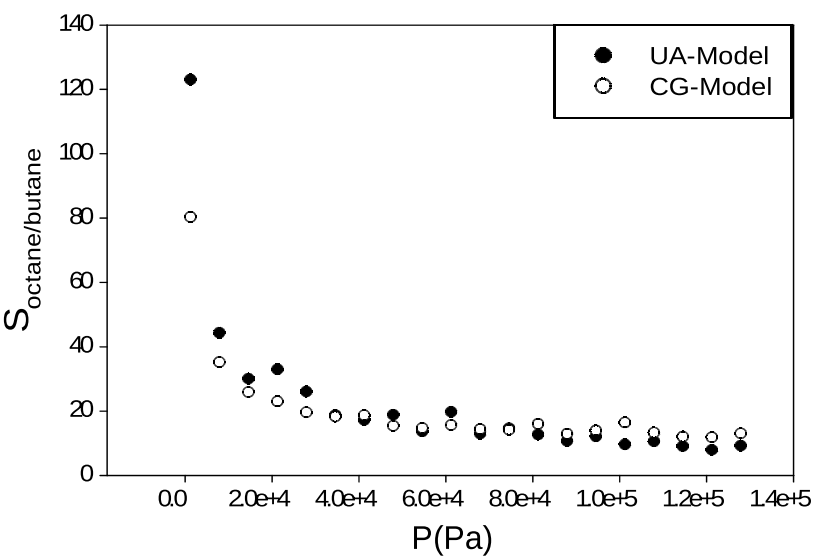

Fig. 8 Selectivity octane-butane

$\mathrm{S}_{\mathrm{ij}}$ is the selectivity, $\mathrm{n}_{\mathrm{i}} / \mathrm{n}_{\mathrm{j}}$ is the number of adsorbed molecules and $\mathrm{x}_{\mathrm{i}} / \mathrm{x}_{\mathrm{j}}$ is the gas phase composition.

The selectivity is shown in Fig. 8 and the coincidence is observed throughout the pressure range, which shows that both models work very well to study the adsorption of mixtures on graphite.

For both models, the degree of separation is greater at low pressures. This is because at these pressures the $n$-butane practically does not adsorb on the surface of the graphite.

\subsection{Adsorption on molecular models of activated carbon}

When studying the adsorption of $n$-butane on models of carbonaceous substrates more complicated as activated carbons, it is found that there is no longer such a good agreement between atomistic models and coarse-grain models (Fig. 9).

This is due to two factors that can be analyzed separately. First, the morphology of the activated carbons causes a distribution of pore sizes, from micropores to macropores, to occur. The pseudo-atoms of $\mathrm{C}$ of the coarse-grain model are 
Fig. 9 Adsorption isotherms for activated carbons
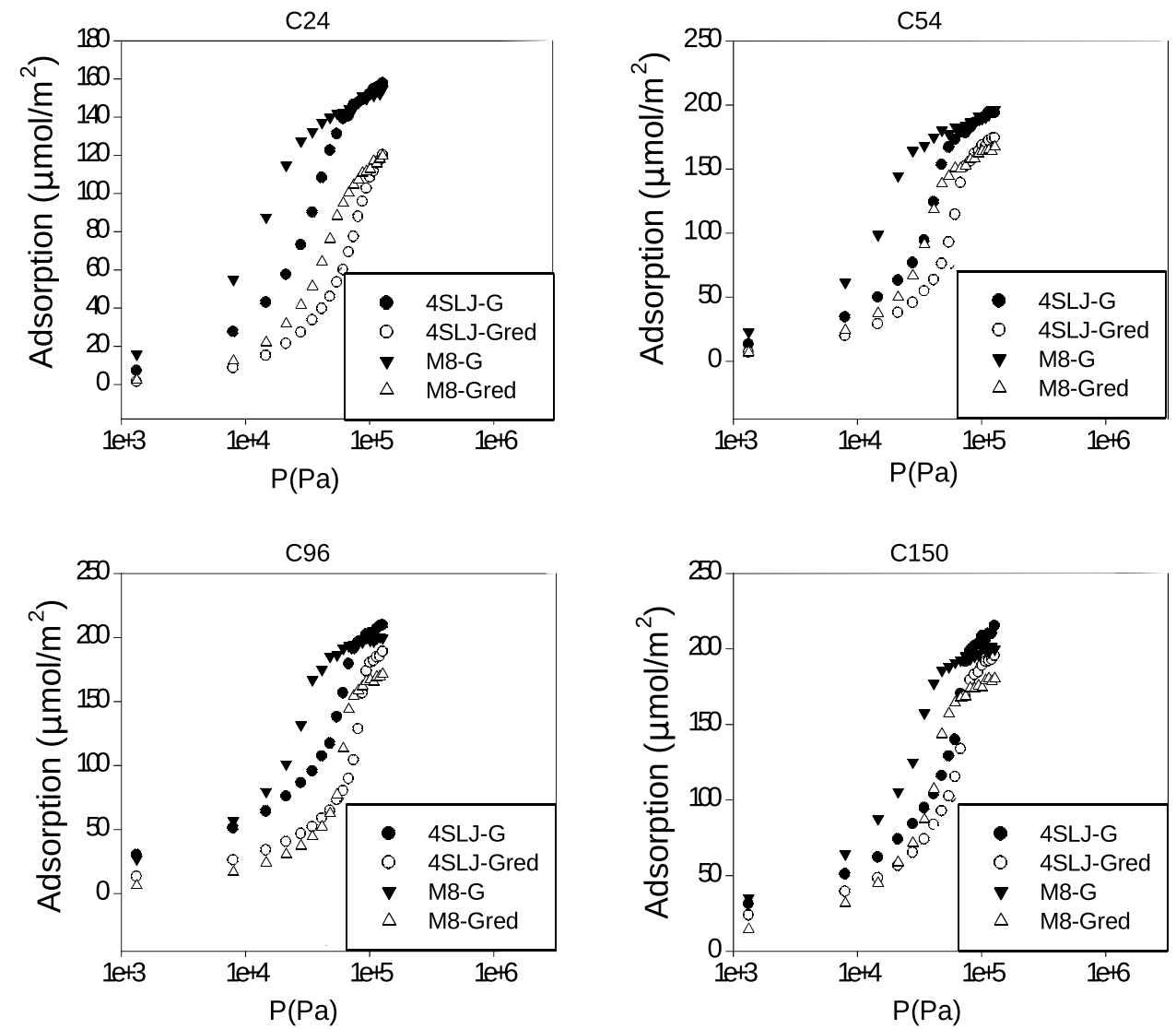

$30 \%$ larger than the atoms of $\mathrm{C}$ of the atomistic model, this generates that, by overlapping, the available volume of the micropores of the coarse-grain model is smaller than in the atomistic model. Consequently, the available place for the adsorption of the molecules is smaller, and the adsorption is lower in the coarse grain model for the $\mathrm{C}$-graphitic.
Fig. 10 Snaphots of a adsorption of 4SLJ-butane on C24 AA, $\mathbf{b}$ adsorption of CG-Butane on C24 CG, $\mathbf{c}$ Adsorption of 4SLJ-butane on C150 AA, $\mathbf{d}$ adsorption of CG-butane on C150 CG
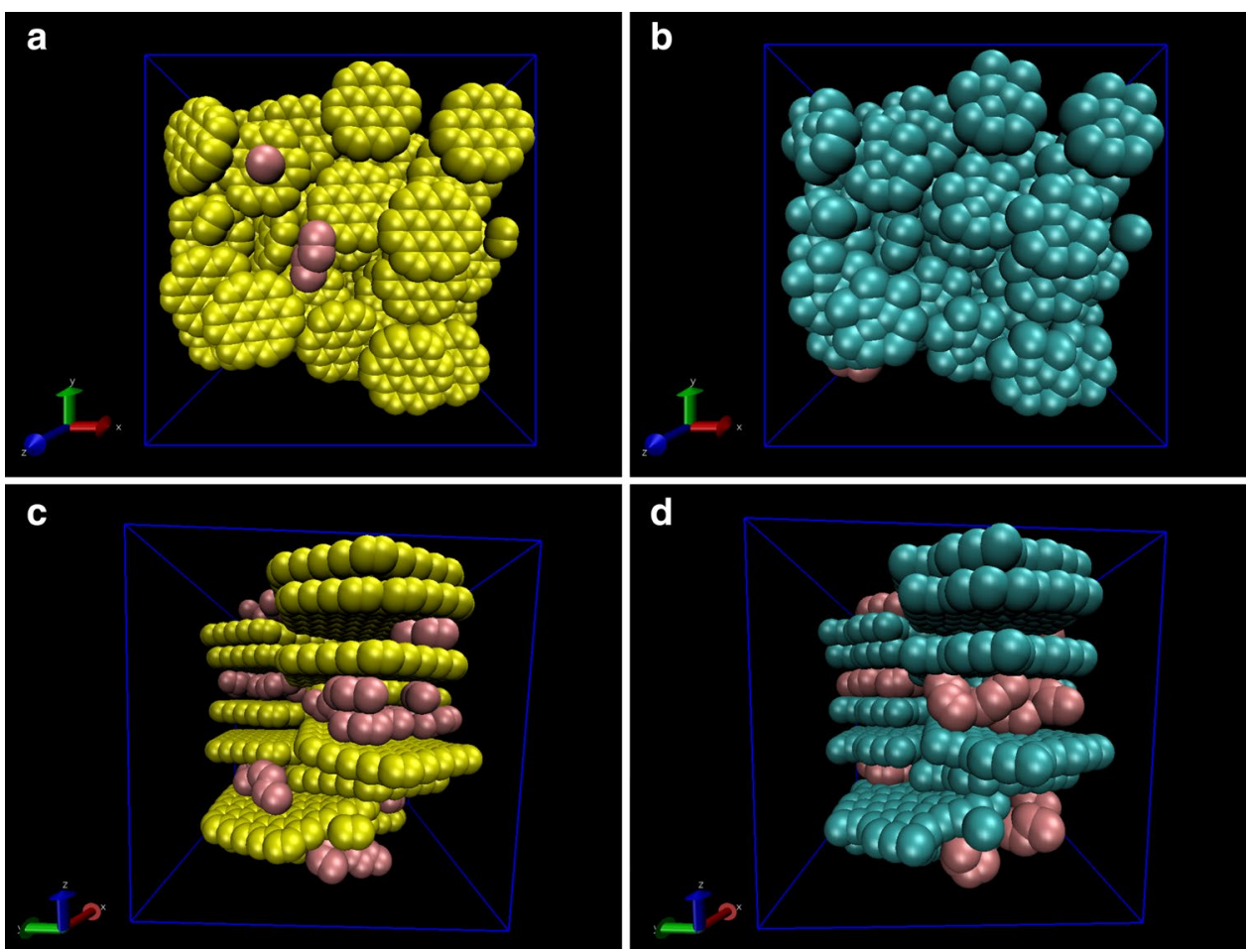
The inverse effect is observed when considering the two models for the molecules that are adsorbed: the coarse grain model, as mentioned before, has a spherical symmetry, which implies that the molecules can be adsorbed in smaller interstices with greater ease than molecules of the atomistic model (Fig. 10).

Being inverse effects, the coarse-grain model for butane molecules, along with the coarse grain model for graphite, works better than when the models are considered separately.

\section{Conclusions}

Our Monte Carlo computer simulations, conducted using a new set of Lennard-Jones parameters, which we termed "mixed" parameters, are able to reproduce both the features of the isotherms (before and after gate-opening) as well as the values of the isosteric heat better than other sets of parameters that had been used in previous simulation studies of sorption in coarse grain simulations. We verified the transferability of this potential by performing calculations for butane and octane with excellent results.

The simulation results for the $n$-alkanes adsorption on graphite, using the CG model, showed an excellent agreement with the atomistic model. However, for the activated carbons studied there is no longer such a good agreement between atomistic models and coarse-grain models, probably due to the porous structure of materials and their smaller porous size.

Acknowledgements We gratefully acknowledge financial support from the UNLP (Universidad Nacional de La Plata) and CONICET (Consejo de Investigaciones Científicas y Tecnológicas).

Funding Funding was provided by Fondo para la Investigación Científica y Tecnológica (Grant No. PICT 2014-3259).

\section{References}

Albesa, A.G.: Simulación Monte Carlo de adsorción de nitrógeno en un modelo molecular decarbón activado y su comparación con resultados experimentales. Avances en Ciencia e Ingeniería 1(2), $47-58(2010)$

Albesa, A.G., Llanos, J.L., Vicente, J.L.: Comparative study of methane adsorption on graphite. Langmuir 24(8), 3836-3840 (2008)

Albesa, A.G., Rafti, M., Rawat, D.S., Vicente, J.L., Migone, A.D.: Ethane/ethylene adsorption on carbon nanotubes: temperature and size effects on separation capacity. Langmuir 28(3), 1824-1832 (2012a)

Albesa, A.G., Rafti, M., Vicente, J.L., Sánchez, H., Húmpola, P.: Adsorption of $\mathrm{CO}_{2} / \mathrm{CH}_{4}$ mixtures in a molecular model of activated carbon through Monte Carlo simulations. Adsorpt. Sci. Technol. 30(8-9), 669-689 (2012b)
Chirnside, G.C., Pope, C.G.: Limiting isosteric heats of adsorption of $n$-butane, $n$-pentane, and $n$-hexane on graphitized carbon black. J. Phys. Chem. 68(8), 2377-2379 (1964)

Chiu, S.W., Scott, H.L., Jakobsson, E.: A coarse-grained model based on Morse potential for water and $n$-alkanes. J. Chem. Theory Comput. 6(3), 851-863 (2010)

Eisen, O.G., Kiselev, A.V., Pilt, A.E., Rang, S.A., Shcherbakova, K.D.: Gas chromatographic investigation of adsorption of normal alkenes C6-C10 on graphitized thermal carbon black. Chromatographia 4(10), 448-454 (1971)

Elkington, P.A., Curthoys, G.: Heats of adsorption on carbon black surfaces. J. Phys. Chem. 73(7), 2321-2326 (1969)

Fletcher, A.J., Thomas, K.M.: Adsorption and desorption kinetics of n-octane and n-nonane vapors on activated carbon. Langmuir 15(20), 6908-6914 (1999)

Fletcher, A.J., Yüzak, Y., Thomas, K.M.: Adsorption and desorption kinetics for hydrophilic and hydrophobic vapors on activated carbon. Carbon 44(5), 989-1004 (2006)

Foley, P., Gonzalez-Flesca, N., Zdanevitch, I., Corish, J.: An investigation of the adsorption of $\mathrm{C} 5-\mathrm{C} 12$ hydrocarbons in the ppmv and ppbv ranges on carbotrap B. Environmental science \& technology 35(8), 1671-1679 (2001)

Gobbo, C., Beurroies, I., de Ridder, D., Eelkema, R., Marrink, S.J., De Feyter, S., de Vries, A.H.: MARTINI model for physisorption of organic molecules on graphite. J. Phys. Chem. C 117(30), $15623-15631$ (2013)

Jiang, C., Ouyang, J., Wang, L., Liu, Q., Li, W.: Coarse graining of the fully atomic methane models to monatomic isotropic models using relative entropy minimization. J. Mol. Liquids 242, 1138-1147 (2017)

Liu, J., Wang, L., Xi, S., Asthagiri, D., Chapman, W.G.: Adsorption and phase behavior of pure/mixed alkanes in nanoslit graphite pores: an iSAFT application. Langmuir 33(42), 11189-11202 (2017)

Maerzke, K.A., Siepmann, J.I.: Transferable potentials for phase equilibria - coarse-grain description for linear alkanes. J. Phys. Chem. B 115(13), 4464-4470 (2001)

Martin, M.G., Siepmann, J.I.: Transferable potentials for phase equilibria. 1. United-atom description of n-alkanes. J. Phys. Chem. B 102(14), 2569-2577 (1998)

Mognetti, B.M., Virnau, P., Yelash, L., Paul, W., Binder, K., Müller, M., MacDowell, L.G.: Coarse-grained models for fluids and their mixtures: comparison of Monte Carlo studies of theirphase behavior with perturbation theory and experiment. J. Chem. Phys. 130(4), 044101 (2009)

Ross, J.W., Good, R.J.: Adsorption and surface diffusion of n-butane on spheron $6\left(2700^{\circ}\right)$ carbon black. J. Am. Chem. Soc. 60, 1167 (1956)

Severson, B.L., Snurr, R.Q.: Monte Carlo simulation of n-alkane adsorption isotherms in carbon slit pores. J. Chem. Phys. 126(13), 134708 (2007)

Shang, J.J., Yang, Q.S., Liu, X.: New coarse-grained model and its implementation in simulations of graphene assemblies. J. Chem. Theory Comput. 13(8), 3706-3714 (2017)

Shelley, J.C., Shelley, M.Y., Reeder, R.C., Bandyopadhyay, S., Klein, M.L.: A coarse grain model for phospholipid simulations. J. Phys. Chem. B 105(13), 4464-4470 (2001)

Publisher's Note Springer Nature remains neutral with regard to jurisdictional claims in published maps and institutional affiliations. 